\title{
Cortical tracking of speech reveals top-down reconstructive processes.
}

Sankar Mukherjee, Alice Tomassini, Leonardo Badino, Aldo Pastore, Luciano Fadiga, Alessandro D’Ausilio

The corresponding author, Sankar Mukherjee, in agreement with all authors, wishes to withdraw the preprint entitled "Cortical tracking of speech reveals top-down reconstructive processes." Upon reviewing the experimental procedure, the authors found fundamental errors undermining the reliability and validity of the reported results. The corresponding author assumes full responsibility for these errors. All authors wish to collect additional data to support the research conclusions. Therefore, the authors do not wish this work to be cited as reference for the project. If you have any questions, please contact the corresponding author. 